\title{
Cooperative Search with Autonomous Vehicles in a 3D Aquatic Testbed
}

\author{
Matthew Keeter ${ }^{1}$, Daniel Moore ${ }^{2,3}$, Ryan Muller ${ }^{2,3}$, Eric Nieters ${ }^{1}$, Jennifer Flenner ${ }^{4,5}$, \\ Susan E. Martonosi ${ }^{2}$, Andrea L. Bertozzi ${ }^{6}$, Allon G. Percus ${ }^{4}$, Rachel Levy ${ }^{2,7}$
}

\begin{abstract}
We consider the problem of cooperative search using autonomous aquatic vehicles, giving a proof-of-concept demonstration in an aquatic testbed. We implement a point-topoint controller for remote-controlled submarines with severe control and buoyancy limitations, develop software to track their motion and establish reliable communication and control. We then propose multiple randomized algorithms, based on Lévy flights, for locating sparse targets in a three-dimensional bounded environment. These algorithms are tested in simulation, showing that they are competitive with nonrandom systematic search, while likely also more robust. The simulations are combined with in-tank tests to display performance under real physical conditions.
\end{abstract}

\section{INTRODUCTION}

While multi-agent coordination for autonomous vehicles has been studied extensively in two-dimensional environments [1], [2], [3], little work has been done on the threedimensional problem. Many applications for autonomous vehicles involve three-dimensional domains, notably aerial and aquatic environments. Such applications include monitoring of atmospheric conditions, surveillance and rescue operations, among others. Three-dimensional environments often impose limits on vehicle mobility, ranging from the non-holonomic constraint of a finite turning radius to the turbulent effects of air and water currents. Implementing control algorithms under these conditions can therefore be a significant challenge.

We consider the problem of cooperative search using autonomous aquatic vehicles. Cooperative underwater robotics experiments are somewhat few and far between, compared to land-based work. Several platforms have been documented in ocean environments [4], [5], in rivers and lakes [6] and in indoor facilities such as swimming pools [7]. Additional examples use surface vehicles such as kayaks [8] or lowspeed air vehicles (e.g., balloons) as proxies for underwater vehicles [9]. Ocean-going vessels can be prohibitively expensive for the university researcher, costing from $\$ 500 \mathrm{~K}$ to over a million dollars for a larger deep-sea vehicle, depending on the desired size, sensors, and depth capabilities. Vehicles on the scale of meters, such as a glider, can still cost on the order of $\$ 100 \mathrm{~K}$ for a single vehicle. A surface kayak, outfitted with

Department of $\left\{{ }^{1}\right.$ Engineering, ${ }^{2}$ Mathematics, ${ }^{3}$ Computer Science $\}$ Harvey Mudd College, 301 Platt Blvd., Claremont, CA 91711

${ }^{4}$ School of Mathematical Sciences, Claremont Graduate University, 710 N. College Ave., Claremont, CA 91711

${ }^{5}$ Naval Air Warfare Center Weapons Division, Weapons and Energetics, China Lake, CA 93555

${ }^{6}$ Department of Mathematics, University of Califonia, Los Angeles, CA 90095.

${ }^{7}$ Contact: levy@math.hmc.edu autonomous controls and sensors may cost on the order of $\$ 20 \mathrm{~K}$. Even in the example with the smallest platform [7], the vehicles span on the order of a meter and the testbed arena has the footprint of a room. Independent of cost, these larger technologies are often prohibitive for smaller campus laboratories due to space constraints. Many groups do not have access to marine resources or even dedicated space with a larger tank or pool. The goal of this work is to build a truly miniaturized aquatic testbed that fits in the space of a large aquarium with robots on the scale of centimeters rather than meters. Such a testbed has the advantage of using cleaner radio signals due to the size of the tank - the challenge is the design and control of the miniature vehicles. Our work parallels a number of prior papers on miniature land-based robots and takes these small robotics experiments to the 3D domain with swimming vehicles. A major challenge of this particular testbed is the underactuation of the vehicles, leading to some redundancy of motion, as discussed in detail in the manuscript.

In this paper, we implement a point-to-point controller, in a testbed tank, for mobile sensors with very limited motion and control capabilities. The robots used are inexpensive, 8 $\mathrm{cm}$ long, remote controlled (RC) submarines. By restricting ourselves to primitive devices, we provide a proof-of-concept demonstration of how such vehicles may effectively perform search functions even under severe control and buoyancy limitations. We use our testbed implementation to investigate three-dimensional search strategies for single and multiple vehicles, extending existing two-dimensional search algorithms [10], [11], [12], [13], [14], [15]. The strategies are based on Lévy flights, in which path directions are chosen uniformly at random but with a path length sampled from a power-law distribution, offering the possibility of occasional long-distance motion. This kind of search is known to be effective for foraging problems and has been documented in several animal behavior studies [16], [11], [14]. We adapt these algorithms to coordinate multiple submarines, combining simulations with actual tank tests to find efficient methods of locating sparse targets in the physical testbed.

Section II presents the testbed hardware and systems. In Section III, we describe single and multi-agent search strategies, while Section IV presents results from largescale simulation. Finally, Section V presents conclusions and suggestions for future work.

\section{TESTBED HARDWARE AND SYSTEMS}

The testbed is a $208 \times 70 \times 40 \mathrm{~cm}$ tank (Fig. 1). Tracking data are collected using a set of cameras, through a pro- 


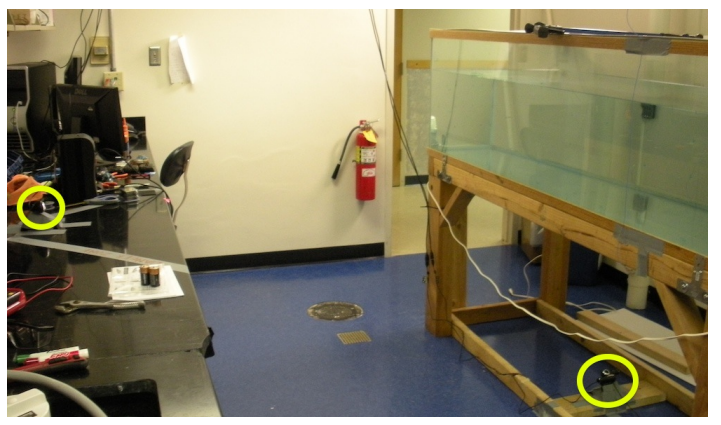

Fig. 1. Camera positions are circled in yellow. One faces the side of the tank, and two view the bottom.

gram that extracts location and rotation. The control system uses the desired locations of each submarine to calculate the necessary motor commands. The commands are then passed through an Arduino microcontroller to the physical submarines. The information flow is shown in Fig. 2.

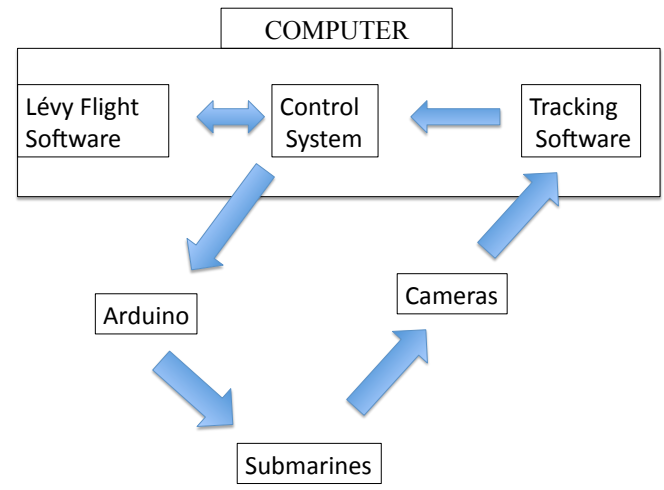

Fig. 2. Simultaneous information flow in the system.

\section{A. Submarines}

The submarines used in this first-generation testbed are $8 \mathrm{~cm} \times 2.6 \mathrm{~cm}$ SUB-SONIC XP recreational class models. They include three propellers. Two rear propellers allow for movement in the horizontal plane, either forward, backwards or off. A central propeller allows for movement along the $z$-axis, with integer settings ranging from -7 (maximum downwards motion) to +7 (maximum upwards motion). There is no built-in mechanism to regulate buoyancy, making motion control particularly challenging.

The vehicles were modified for better control and tracking. To track orientation, colored tape was placed to the bottom of each submarine, with a different color at the bow and stern. For better control, resistors (between 2.7 and $5.6 \Omega$ ) were soldered into the wires leading to the motors, beneath the plastic cases, resulting in slower but more controlled actuation. Finally, to achieve neutral buoyancy, they were weighed down with coils of lead wire and styrofoam pieces. The modifications led to some unavoidable leaking, and thus to increasingly negative buoyancy over the course of an experiment.

\section{B. Communication}

The remote controls included with the original toy submarines were disassembled, and wires were soldered into the printed circuit board at button terminals. This electrically mimics the thumbs of a human operator. A similar strategy was used in a first generation land-based miniaturized testbed [17]. These wires were connected to an Arduino microcontroller, which was in turn connected to a computer. The Arduino, running custom software, receives commands on its serial input, then translates these to the appropriate electrical impulses.

\section{Tracking}

Given the small size of our submarines, it was infeasible to embed on-board sensors. Instead, we developed a tracking system, building on the work by Pruitt et at. [18]. The tracking system is implemented in C++ and OpenCV [19]. Three cameras image the tank, as shown in Figure 1: two from the bottom, each of which view about half of the tank, and one from the side. The bottom camera images are dekeystoned, to correct for distortion from the camera angles, and then stitched together. The stitched bottom image and the side image are then filtered in a similar process. A subimage of each known submarine is extracted, as shown in Figure 3. The algorithm for the bottom cameras also takes color into account when generating subimages, inferring submarine orientation by drawing a straight line between the most likely bow and stern subimages. Finally, a precomputed transformation converts pixel location ( $x$ and $y$ location in bottom and side images) to physical location ( $x$, $y$, and $z$ coordinates relative to the corner of the tank). This transformation was computed using data from calibration images using a quadratic fit.

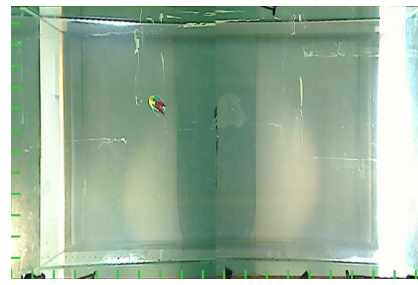

(a)

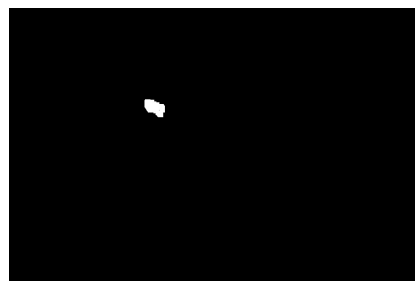

(c)

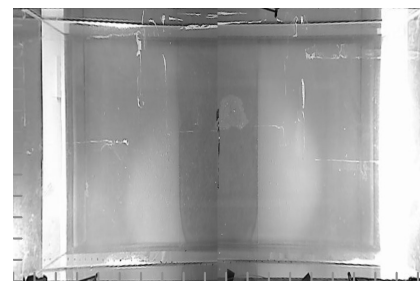

(b)

(d)

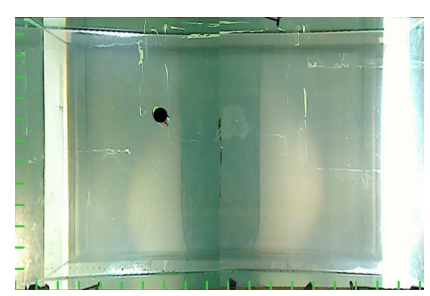

Fig. 3. Starting from the stitched image (a), a previously captured image of an empty tank (b) is subtracted. The resulting image is dilated, smoothed and thresholded (c) to produce a subimage. Using the CvBlob library within OpenCV, the new subimage is located (d) in the frame. 


\section{Control}

Due to the primitive steering mechanism of the robot, we implemented a bang-bang type controller for point-topoint control, involving separate processes for horizontal and vertical motion. The horizontal location control process relies on the angle between the horizontal heading of the submarine and the direction of the target point (Fig. 4). One of five fixed maneuvers is implemented depending on this heading:

- If the destination is directly ahead (within a threshold angle $\alpha_{1}$ ), go forward with both motors.

- If the destination is between $\alpha_{1}$ and a second threshold angle $\alpha_{2}$ (in either direction), go forward with one motor while keeping the other turned off. This results in a modest turn towards the target and forward motion.

- If the destination is greater than the threshold angle $\alpha_{2}$ to the right or left, go forward with one motor and backwards with the other motor. This will cause a fast rotation towards the target and very little forward/backward motion.

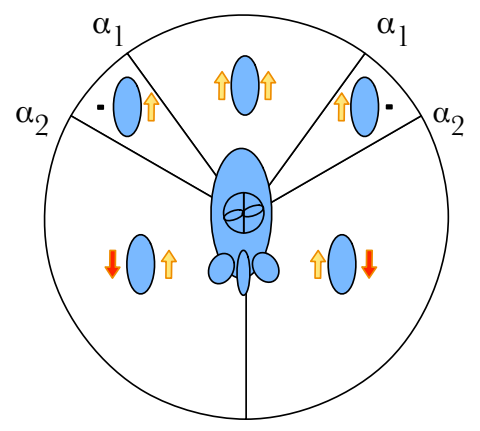

Fig. 4. Left and right motor behavior as a function of destination's angular position. Arrows indicate motion direction and a dot indicates no motor action. Values $\alpha_{1}$ and $\alpha_{2}$ are thresholds delimiting each type of movement.

The threshold angles $\alpha_{1}$ and $\alpha_{2}$ are adjustable parameters, currently set at $\alpha_{1}=\frac{\pi}{5}$ and $\alpha_{2}=\frac{\pi}{3}$. These choices provide sufficient horizontal control, although they could presumably be optimized further. The vertical control is a simple bangbang control. If the submarine is below the destination, it activates the motor to send it upwards; if above, the motor is activated to send it downwards. In order to avoid the motion instabilities that arise from using both horizontal and vertical control processes simultaneously, we toggle between the two, using the following strategy. First, adjust the vehicle's vertical location, without moving horizontally, until it is within $2.5 \mathrm{~cm}$ of the target's vertical location. Then switch to horizontal control, moving the vehicle towards the target with its vertical propeller stopped. If at any point its vertical position is more than $10 \mathrm{~cm}$ away from the target's vertical position, restart the vertical location adjustment. This strategy was implemented as a state machine in the MATLAB code for the control system.

\section{E. In-Tank Test}

To examine the precision of the control system, we examined the results of a single jump, from a known starting point. The submarine started on a simple launch platform and was directed to a point on the other side of the tank using the automated control system. Two examples of this single jump are showed in Figure 5. In spite of the severe buoyancy and mobility challenges discussed earlier, the submarines were successful in navigating autonomously from the launch platform to the desired destination. Note that the bang-bang style controller results in oscillatory motion in both the horizontal and vertical directions, however the robot still reaches its destination. This type of motion is typical of underactuated controllers such as those sometimes used to steer a ship. In [20] a time-periodic underactuated controller was designed, however the system was assumed to have relatively small noise resulting in the design of a smooth (sinusoidal) time-periodic controller rather than the bangbang controller used for our primitive submarines.

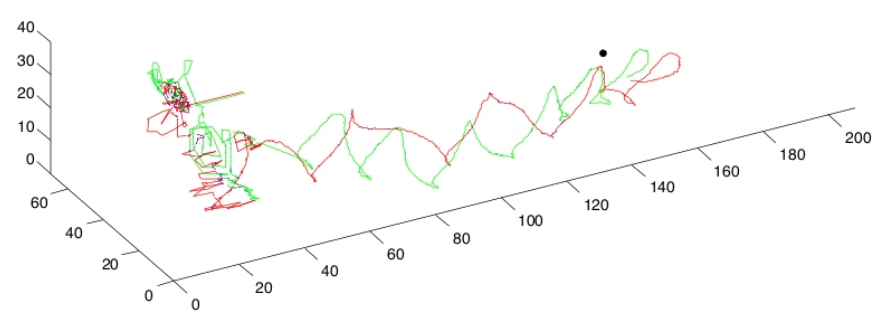

Fig. 5. Two single-jump tests, superimposed in different colors. All tests start on the launch platform and travel towards the point marked with a black dot. The control system results in similar paths for the two separate runs.

\section{SEARCH ALGORITHMS AND SIMULATIONS}

A practical application of this simple physical testbed is target searching in a three-dimensional aquatic environment. Many search problems in real-world environments of this kind, such as finding animals for tagging, lost boats, or mines in minefields, involve sparsely located targets. These applications are best modeled with a destructive target scheme in which targets may only be found once. Previous studies have found the use of Lévy flights to be an efficient strategy for finding sparse targets in a two-dimensional environment [10], [11], [12], [13], [14], [15], and more recent results suggest that this may apply in three dimensions as well [21]. In what follows, we simulate and implement several methods for target search using aquatic vehicles that follow Lévy flights.

\section{A. Lévy Flight}

A Lévy flight is a random walk with step lengths chosen from a heavy-tailed probability distribution. Steps in the three-dimensional Lévy flight are constructed as follows. First, a point is chosen uniformly at random on the surface of a unit sphere. This establishes the direction for the step. Second, the step length $\ell$ is chosen from a probability density

$$
f(\ell)=x_{0}^{\lambda-1}(\lambda-1) \ell^{-\lambda}
$$


for $\ell \geq x_{0}$, where $x_{0}$ is the minimum step length, and $\lambda$ is known as the Lévy exponent. This distribution can be normalized provided that $\lambda>1$. For $1 \leq \lambda \leq 3$, the step length has infinite variance, resulting in a path that mixes short and long jumps: $\ell$ has a scale-free distribution, though smaller values of $\lambda$ will generate long jumps more frequently than larger values. For $\lambda>3$, the variance is finite, and the central limit theorem applies, resulting in Brownian motion with a fixed diffusion length-scale determined by the variance. Intuitively, Lévy flights with $\lambda \leq 3$ are effective search strategies because the scale-free distribution occasionally causes an unusually long jump. Thus, the search agent thoroughly searches a small region of the space, then jumps to a region that is likely previously unexplored and begins again. This behavior is similar to that of a foraging animal who searches for food in a given area with a series of small movements, then travels a larger distance to another area to search again.

In our search algorithm, we consider a target to be discovered if the searching vehicle passes sufficiently close to it. Some groups have investigated target detection with noisy sensors, so that there is no guarantee of finding a nearby target [3], but we will assume for simplicity that any target within a given detection radius is discovered. Using the destructive target scheme, we count each unique target and continue the search until a predetermined number of targets are discovered.

\section{B. Multi-agent Search Strategies}

We have considered four different Lévy search strategies for multiple agents.

1) Independent search: Each submarine independently runs a Lévy search in the entire tank.

2) Bounded region search: Divide the tank into regions. Each submarine is assigned to a region and runs a bounded Lévy search that only returns target points in that region. This method is also known as "divide-andconquer" [3].

3) Biased angle search: A submarine biases its choice of direction based on the position of other submarines. The direction is chosen from a von Mises-Fisher distribution [22]

$$
f(\theta) \propto e^{\kappa \cos \theta},
$$

which is close to a normal distribution on a circle. This distribution has previously been used to bias a Lévy walk when a priori information is known about the target distribution [12]. In our case, we define $\theta=0$ to be the direction opposite that of the nearest other submarine. The parameter $\kappa$ approximates the reciprocal of the distribution's variance, so that submarines repel each other with high probability when $\kappa$ is large, whereas there is no angular bias when $\kappa=0$. In order to set $\kappa$ for a given vehicle, we assume that the distance $d$ to the nearest other vehicle is known: in our testbed, position information is passed through the Arduino microcontroller, though in a real setting it would be determined by direct inter-vehicle broadcast. We then let $\kappa=d_{0} / d$. Empirically, we have found that the algorithm's performance is best when $d_{0}$ is close to the detection radius, though it is relatively insensitive to its exact value. For simplicity, we set $d_{0}$ to exactly the detection radius.

4) Biased jump length search: A submarine dynamically chooses a value of $\lambda$ for its jump length distribution. Prior results have shown that $\lambda$ should approach 1 for optimal search [13]. Therefore, we vary $\lambda$ towards 1 when other submarines are nearby-they are more likely to take long jumps - and towards 2 otherwisethey mostly take short jumps. We let each submarine adjust $\lambda$ to be

$$
\lambda=1+e^{-\left(d_{1} / d\right)^{2}}
$$

where $d$ is again the distance to the nearest other vehicle. We set the parameter value $d_{1}=100 \mathrm{~cm}$, so that $\lambda=1.5$ when the two submarines are approximately half of the maximal distance from each other in the tank.

\section{Implementation}

In order to determine the optimal parameter(s) for each multi-agent search strategy and the best such strategy overall, we implemented a simulation framework in MATLAB with the following settings. The search space dimensions were chosen to be $x \times y \times z=208 \times 70 \times 40 \mathrm{~cm}$. For the bounded region search for two submarines, we divide the tank into two halves by cutting across the $x$ dimension. The simulated detection radius is $r=10 \mathrm{~cm}$. The minimum jump length is $x_{0}=5 \mathrm{~cm}$. For path planning purposes we assume reflective boundaries. The search space contains four targets, with the same set of targets used in every run for every simulation type: two distinct targets must be found for the search to complete. For each of the algorithms (excluding the biased jump length algorithm), we varied the value of $\lambda$ between 1.1 and 3.1 in increments of 0.5. Each setting was run 5,000 times in simulation. Performance is measured by the total path length, which is indicative of the total time spent searching. When there are multiple submarines, the path length is measured for the submarine that finds the second unique target, up to the jump in which it finds that target.

\section{SIMULATION RESULTS}

\section{A. One Vehicle}

In all algorithms, performance is best as $\lambda$ approaches 1 . The quality degrades as $\lambda$ increases, continuing to do so for $\lambda>3$ as the motion becomes Brownian with decreasing variance. Thus, frequent long jumps are clearly favorable. At $\lambda=1.1$, where performance is best among the values that we implemented, the average search length for two vehicles performing a bounded region search $(693 \mathrm{~cm})$ is almost exactly half that of a single vehicle $(1388 \mathrm{~cm})$, as one might ideally expect. Interestingly, as $\lambda$ increases, the performance of a single submarine degrades faster than that 


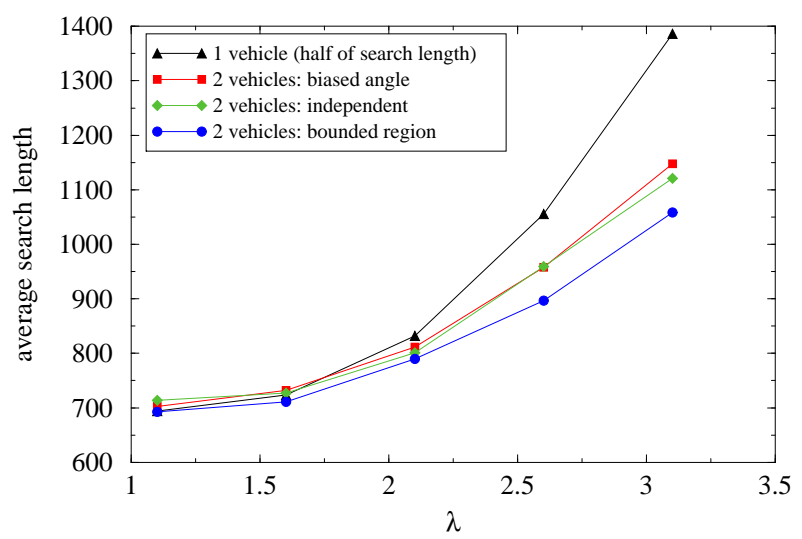

Fig. 6. Results from five thousand simulations showing average search length vs. $\lambda$. For ease of comparison, the single-vehicle results are for half of the search length. Best performance is found at $\lambda=1.1$, consistent with existing two-dimensional results [10]. The biased jump length algorithm sets $\lambda$ dynamically: these results are shown in Figure 7.

of the two-submarine search methods. This is consistent with the intuition that the longer jumps are crucial for searching in a larger region such as the entire tank. The results are shown in Figure 6, which compares half of the single-vehicle search length with the two-vehicle search lengths.

\section{B. Two Vehicles}

Figure 7 compares the results for two submarines at $\lambda=$ 1.1 , showing the $95 \%$ confidence intervals for search distance under the four different strategies.

Two submarines implementing the bounded region (divide-and-conquer) strategy had the lowest average search length, consistent with prior results in two dimensions [3]. The method was implemented by dividing the tank into two equal halves along the $y z$-plane. The fact that the searchers only move within their own region means that they do not waste time overlapping.

The average search length for the biased angle algorithm was about $1 \%$ higher than for the bounded region algorithm at $\lambda=1.1$. Given the confidence intervals, this difference is not statistically significant. In our tests, the performance of the biased angle algorithm appeared relatively insensitive to the exact value of the parameter $d_{0}$ (and hence $\kappa$ ). However, these results suggest that with a more exhaustive study, the method could conceivably be tuned to the point where it outperforms the other search algorithms. Note that for $d_{0}$ too small, $\kappa$ will be small and the variance in angle will be large, so the method will not differ from independent search. For $d_{0}$ too large, the submarines will repel each other and therefore avoid the center of the tank.

The biased jump length algorithm performed the worst, compared with the other strategies with fixed $\lambda=1.1$. Determining the best value of the parameter $d_{1}$ (and hence the dynamic $\lambda$ ) for this algorithm is again a delicate balancing act. The average distance between vehicles in this search seems greater than reasonable, meaning that as in the biased angle algorithm with large $d_{0}$, submarines are not spending enough time in the center of the tank. The performance can

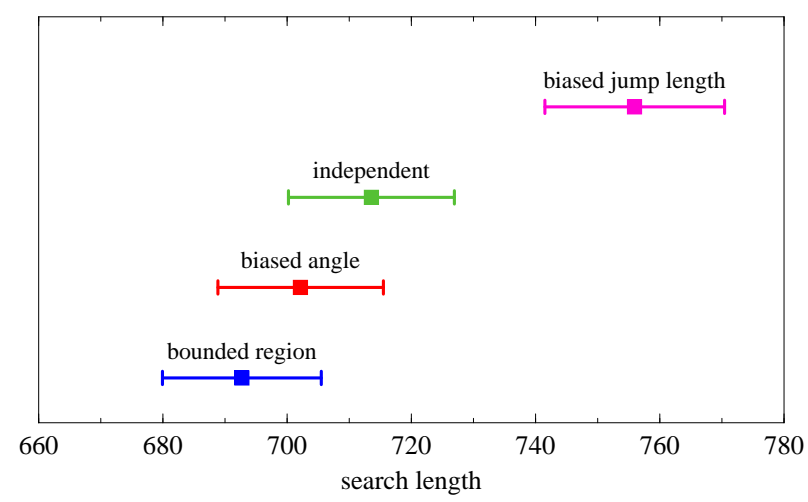

Fig. 7. Statistical comparison of algorithms for two submarines at $\lambda=1.1$, showing average search length and $95 \%$ confidence intervals. Differences in performance of bounded region (divide-and-conquer), biased angle and independent methods are not statistically significant. Biased jump length, where $\lambda$ is set dynamically, performs poorly compared to the others at $\lambda=1.1$.

be improved by increasing $d_{1}$ further, so as to make larger values of $\lambda$ more rare. However, whether those large values of $\lambda$ are ever helpful is inconclusive.

\section{Comparison with Systematic Search}

It is instructive to compare these simulation results with what one would theoretically expect from a systematic (raster scan) search of the tank. Comparisons of this sort have previously been performed in one and two dimensions [23], [24]. These studies confirmed that in the absence of any a priori knowledge of the target distribution, one cannot generally outperform a perfectly executed raster scan search given a sufficiently large search space. A lower bound for the travel distance required to search the entire tank with a raster scan search is calculated as follows. Consider the minimal distance swept out by parallel but overlapping cylinders, oriented along the $x$ dimension of the tank, such that in the cross-sectional $y z$-plane these cylinders cover the entire tank. Figure 8 shows that 15 cylinders are needed, given the $y$ - and $z$-dimensions of our tank. Since the tank's length is $x=208 \mathrm{~cm}$, the length of each cylinder must be at least $x-2 r / \sqrt{2}=208-10 \sqrt{2} \mathrm{~cm}$, accounting for the additional hemispherical region detected at the two ends of the cylinder. This gives a lower bound on the length of the raster scan path of $15 \times(208-10 \sqrt{2}) \approx 2908 \mathrm{~cm}$. Thus, the average search length for a single raster search to find half of all targets ( 2 out of 4 ) distributed uniformly at random in the tank is bounded below by half of this total distance, or $1454 \mathrm{~cm}$.

It may seem surprising that the single Lévy search vehicle requires only $1388 \mathrm{~cm}$, a $5 \%$ reduction over the apparent lower bound on the raster search. This is a simple consequence of the raster requiring an integer number of cylindrical sweeps. If the search space is increased with the detection radius held constant, the constraint of an integer number of cylinders will become asymptotically negligible, and the path length will be approximately

$$
d \approx \frac{y}{\sqrt{2} r} \frac{z}{\sqrt{2} r}(x-\sqrt{2} r)=\frac{x y z}{2 r^{2}}-\frac{y z}{\sqrt{2} r} .
$$




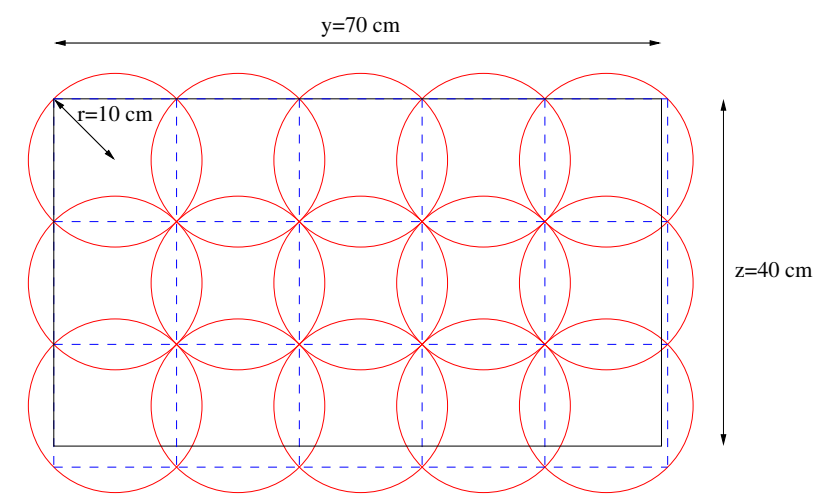

Fig. 8. Cross section in $y z$-plane of cylindrical regions detected by a raster search. For complete coverage of cross-sectional area, centers are placed $\sqrt{2} r=10 \sqrt{2} \mathrm{~cm}$ from each other, requiring at least 5 cylinders in $y$-direction and 3 cylinders in $z$-direction.

Under idealized conditions, this would lead to an approximated average search distance of $1357 \mathrm{~cm}$, which is $2 \%$ below the single Lévy search vehicle distance. However, this assumes that the raster search can be executed perfectly, which is highly unlikely in real aquatic conditions. The Lévy search, by contrast, is likely to be far more tolerant of imprecision because it is in itself a random construction. Thus, even in near-asymptotic conditions, it is not implausible that the Lévy search would slightly outperform the raster scan.

\section{In-Tank Test}

As a proof of concept, we tested the Lévy flight in our physical testbed. This test used the same path generation code as in the simulations, seeded with the same value for every run. Figure 9 plots the submarine's actual motion, as measured from data saved by the tracking system, against its prescribed path.

The aquatic vehicles employed in the experiments were tiny, low-cost $(\$ 40)$ toy submarines. While the tests provided evidence that low-cost hardware could be used, we have determined that for future testing, we will move to a still inexpensive (\$125) but larger and more stable vehicle.

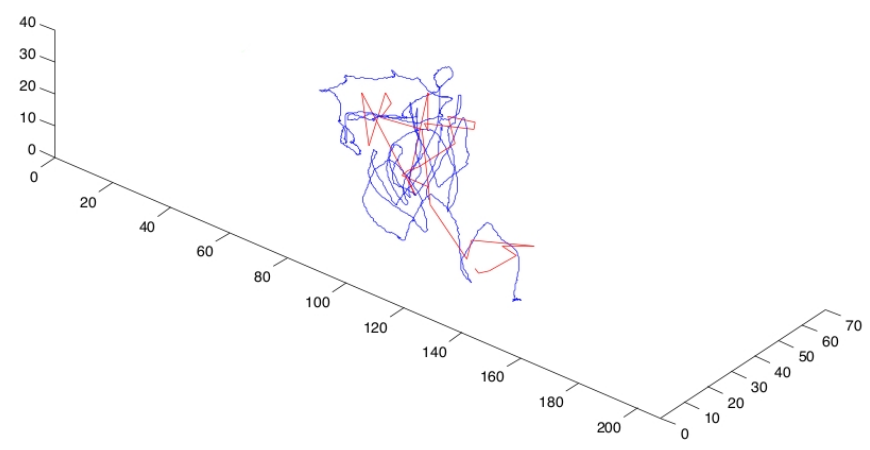

Fig. 9. Typical Lévy search without targets. Path of $955 \mathrm{~cm}$ is two orders of magnitude greater than the length of the vehicle and is limited by buoyancy and battery time. Red path depicts calculated Lévy flight trajectory. Blue path depicts submarine's actual trajectory.

\section{CONCLUSIONS AND FUTURE WORK}

We have provided a proof-of-concept demonstration of cooperative search algorithms for aquatic vehicles with very limited control capabilities. Using inexpensive remote controlled (RC) submarines, we have developed a point-topoint controller that functions even under poorly regulated buoyancy and mobility conditions. We have tested a number of different three-dimensional random search algorithms, based on Lévy flights, for finding sparse targets using these submarines. Our algorithms perform optimally when the Lévy exponent, specifying the degree of the power-law distribution for step lengths, approaches 1. This mirrors earlier findings for Lévy search in two dimensions [10].

We have found in simulations that for a Lévy search with two submarines, bounding the allowable search region for each submarine (divide-and-conquer) results in essentially ideal performance, maintaining almost the identical cumulative search distance and thus reducing search time by $50 \%$. To within statistical error, this performance is comparable to that of the biased angle method that repels nearby vehicles, as well as to that of the independent search method.

Remarkably, a Lévy search outperforms a systematic raster sweep of the entire tank: a single submarine requires an average Lévy search distance that is $5 \%$ below a theoretical lower bound on the raster search distance. This is partly due to finite-size effects in the tank. The raster search requires an integer number of sweeps, resulting in a loss of efficiency for small volumes. However, our results suggest that even under asymptotic (large volume) conditions, the Lévy search will be competitive with raster search. We would expect that the randomness inherent in the Lévy search would make it more robust to control imprecision than the raster search, which requires perfect execution with precise sharp turns in order to maintain its performance quality. Consequently, under imperfect experimental conditions or environmental disturbances, the three-dimensional Lévy search may significantly outperform systematic search methods. Quantifying this effect remains an open problem. Drift and sensor positioning errors may be modeled, for example, by Brownian motion. While ocean currents may induce a directional bias, a simple unbiased model would likely provide a lower bound on the error in raster search. This approach, along with more detailed in-tank comparisons, could help clarify the comparative advantages of Lévy search.

Another direction for further research is the extension of our study to more than two vehicles. This is likely to require a larger physical testbed and a more sophisticated tracking system. Additionally, a second-generation testbed might involve submarines with somewhat better buoyancy control. While there are merits to using imperfect devices in order to create control challenges, the search distance in current in-tank tests may be excessively attributable to buoyancy correction, and make it difficult to perform a true physical testbed analysis of Lévy search algorithms. One option is to use submarines with a ballast system, rather than propellers: preliminary tests with the Graupner U-16 
model [25] show promise. An interesting problem would then consist of optimally designing a controller for these secondgeneration vehicles, based on their own motion limitations.

\section{ACKNOWLEDGMENTS}

This research is supported by Office of Naval Research grant N000141010641, NSF grant EFRI-1024765, the DYNAR research group, and Claremont Graduate University's BLAIS Foundation.

\section{REFERENCES}

[1] T. Chung, L. Cremean, W. B. Dunbar, Z. Jin, E., D. Moore, A. Tiwari, D. V. Gogh, and S. Waydo, "A platform for cooperative and coordinated control of multiple vehicles: The Caltech multi-vehicle wireless testbed," in Proceedings of the 3rd Conference on Cooperative Control and Optimization, 2002.

[2] K. K. Leung, C. H. Hsieh, Y. R. Huang, A. Joshi, V. Voroninski, and A. L. Bertozzi, "A second generation micro-vehicle testbed for cooperative control and sensing strategies," in Proc. Am. Contr. Conf., 2007.

[3] W. Liu, M. Short, A. L. Bertozzi, and Y. Taima, "Multi-Scale collaborative searching through swarming," in Proc. ICINCO, 2010.

[4] N. E. Leonard, D. A. Paley, R. E. Davis, D. M. Fratantoni, F. Lekien, and F. Zhang, "Coordinated control of an underwater glider fleet in an adaptive ocean sampling field experiment in Monterey Bay," Journal of Field Robotics, vol. 27, no. 6, pp. 718-740, 2010.

[5] H. C. Brown, A. Kim, and R. M. Eustice, "An overview of autonomous underwater vehicle research and testbed at PeRL," Marine Technology Society Journal, vol. 43, no. 2, pp. 33-47, 2009.

[6] B. Schulz, B. Hobson, M. Kemp, J. Meyer, R. Moody, H. Pinnix, and M. St Clair, "Field results of multi-UUV missions using ranger micro-UUVs," in OCEANS 2003. Proceedings, vol. 2, Sept. 2003, pp. 956-961.

[7] N. Sydney, S. Napora, S. Beal, P. Mohl, P. Nolan, S. Sherman, A. Leishman, and S. Butail, "A micro-uuv testbed for bio-inspired motion coordination," Proc. Unmanned Untethered Submersible Technologies, 2009, durham, NH.

[8] F. Arrichiello, H. Heidarsson, S. Chiaverini, and G. Sukhatme, "Cooperative caging using autonomous aquatic surface vehicles," in Proc. ICRA, 2010, pp. 4763-4769.

[9] E. Honary, F. Mcquade, R. Ward, I. Woodrow, A. Shaw, D. Barnes, and M. Fyfe, "Robotic experiments with cooperative aerobots and underwater swarms," Robotica, vol. 27, pp. 37-49, January 2009.

[10] G. M. Viswanathan, V. Afanasyev, S. V. Buldyrev, S. Havlin, M. da Luz, E. Raposo, and H. E. Stanley, "Lévy flights in random searches," Phys. A, vol. 282, no. 1-2, pp. 1-12, 2000.

[11] F. Bartumeus, J. Catalan, U. L. Fulco, M. L. Lyra, and G. M. Viswanathan, "Optimizing the encounter rate in biological interactions: Lévy versus Brownian strategies," Phys. Rev. Lett., vol. 88, 2002.

[12] D. Marthaler, A. L. Bertozzi, and I. Schwartz, "Lévy searches based on a priori information: The biased Lévy walk," UCLA CAM Report,(0450), 2004.

[13] A. Reynolds, "Cooperative random Lévy flight searches and the flight patterns of honeybees," Phys. Lett. A, vol. 354, no. 5-6, pp. 384-388, 2006.

[14] A. M. Edwards, R. A. Phillips, N. W. Watkins, M. P. Freeman, E. J. Murphy, V. Afanasyev, S. V. Buldyrev, M. da Luz, E. Raposo, H. E. Stanley, and G. M. Viswanathan, "Revisiting Lévy flight search patterns of wandering albatrosses, bumblebees and deer," Nature, vol. 449, no. 7165, pp. 1044-1048, 2007.

[15] M. Ghaemi, Z. Zabihinpour, and Y. Asgari, "Computer simulation study of the Lévy flight process," Phys. A, vol. 388, no. 8, pp. 1509$1514,2009$.

[16] G. Viswanathan, V. Afanasyev, S. Buldyrev, E. Murphy, P. Prince, and H. Stanley, "Lévy flight search patterns of wandering albatrosses," Nature, vol. 381, no. 6581, pp. 413-415, 1996.

[17] C. Hsieh, Y. Chuang, Y. Huang, K. Leung, A. L. Bertozzi, and E. Frazzoli, "An economical micro-car testbed for validation of cooperative control strategies," in Am. Contr. Conf., 2006, 2006, p. 6 pp.

[18] P. Pruitt, G. Dinolov, A. McAuley, W. Ferenc, A. L. Bertozzi, and R. Levy, "An economical micro-submarine testbed for validation of 3D cooperative control strategies for underwater robots," 2010.
[19] OpenCV, “Open source computer vision," 2010. [Online]. Available: http://opencv.willowgarage.com/wiki

[20] K. Y. Pettersen and T. I. Fossen, "Underactuated dynamic positioning of a shipexperimental results," IEEE Trans. Contr. Sys. Tech., vol. 8, no. 5, pp. 856-863, 2000.

[21] D. Sutantyo, S. Kernbach, P. Levi, and V. Nepomnyashchikh, "MultiRobot searching algorithm using Lévy flight and artificial potential field," in IEEE International Workshop on Safety, Security and Rescue Robotics 2010, 2010.

[22] S. Jung, "Generating von Mises Fisher distribution on the unit sphere $\left(s^{2}\right)$," http://www.unc.edu/ sungkyu/manifolds/randvonMisesFisher3.pdf, 2006, [Online; accessed 3-April-2011].

[23] O. Bénichou, M. Coppey, M. Moreau, and R. Voituriez, "Intermittent search strategies: When losing time becomes efficient," Europhys. Lett., vol. 75, no. 2-6, pp. 349-354, 2006.

[24] O. Bénichou, C. Loverdeau, M. Moreau, and R. Voituriez, "A minimal model of intermittent search in dimension two," J. Phys.: Condensed Matter, vol. 19, p. 065141, 2007.

[25] Graupner, "RC mini-submarine GRAUPNER U-16," http://www.graupner.de/en/products/e8dfd79b-d676-4e4d-b5c42bb59b2b7155/2018/product.aspx, 2011. 